\title{
Junge Menschen und ihre Familien umfassend fördern
}

\section{Zur aktuellen Situation kommunaler Jugendhilfe in Deutschland}

\author{
Reinhard Wiesner
}

Ministerialrat Prof. Dr. Dr.h.c Reinhard Wiesner ist Leiter des Referates Kinderund Jugendhilferechts im Bundesministerium für Familie, Senioren, Frauen und Jugend und Mitglied im Beirat der Blätter der Wohlfahrtspflege. E-Mail reinhard.wiesner@bmfsfj.bund.de
Die Jugendhilfe in Deutschland wird zuallererst kommunal organisiert und finanziert. Auch deshalb ist die Jugendhilfe von Gemeinde zu Gemeinde, von Landkreis zu Landkreis höchst unterschiedlich ausgestaltet, was sich auch in den beiden Arbeitsfeldern der Jugendhilfe zeigt, die seit einiger Zeit im Mittelpunkt der öffentlichen Aufmerksamkeit stehen: die Tagesbetreuung für Kinder und der Kinderschutz.

Jugendhilfe in Deutschland ist zuallererst kommunale Jugendhilfe. Sie gehört zu den traditionellen Bereichen kommunaler Selbstverwaltung. Zwar haben der Bund durch das Kinder- und Jugendhilfegesetz (SGB VIII) und die Länder durch ihre Ausführungsgesetze der kommunalen Selbstverwaltung im Bereich der Jugendhilfe einen Rahmen gegeben.

Dennoch bleiben beträchtliche Gestaltungsspielräume, die örtlich und regional (sehr) unterschiedlich ausgefüllt werden. Hinzu kommt die Kooperation zwischen der öffentlichen und der freien Jugendhilfe als Spezifikum der Jugendhilfe in Deutschland. Deshalb gehört auch die Tätigkeit der freien Hilfe im weiteren Sinn zur kommunalen Jugendhilfe, bleiben doch die Gesamtverantwortung und die Finanzierungslast bei den Kreisen und Städten als örtlichen Trägern der Jugendhilfe. Sie tragen nach der Finanzstatistik 70 Prozent der Ausgaben (die Länder $29 \%$, der Bund 1\%).

Maßgebende Faktoren, die auf die Situation kommunaler Jugendhilfe in Deutschland strukturell und aktuell Einfluss nehmen, sind

- die Situation kommunaler Haushalte

- der Stellenwert der Kinder- und Jugendhilfe im Kontext anderer kommunaler Aufgaben

- die Art und Weise der Aufgabenwahrnehmung im örtlichen Bereich

- die begrenzte Steuerungswirkung bundesgesetzlicher Vorgaben und

- die aktuellen bundesgesetzlichen Schwerpunkte und Herausforderungen innerhalb des komplexen und heterogenen Aufgabenfelds der Kinder- und Jugendhilfe.

\section{Die Situation \\ kommunaler Haushalte}

Zwar hat sich die Situation kommunaler Haushalte aufgrund der konjunkturellen Entwicklung in den letzten Jahren entspannt. Diese Verbesserung wirkt sich indes nicht gleichmäßig in der Fläche aus. Der Stadt Düsseldorf, die damit wirbt, einen schuldenfreien Haushalt verabschiedet zu haben, stehen Städte und Regionen gegenüber, in denen nach wie vor der kommunale Haushalt von staatlicher Seite genehmigt werden muss und kein Spielraum für neue Entwicklungen besteht. Im Kontext der Kinder- und Jugendhilfe ist diese Situation deshalb besonders fatal, weil in denjenigen kommunalen Gebietskörperschaften, die aufgrund struktureller Schwächen und hoher Arbeitslosigkeit sparen müssen, gleichzeitig die materiellen und immateriellen Hilfebedarfe für Kinder, Jugendliche und Familien besonders hoch sind.

\section{Der Stellenwert der Kinder- und Jugendhilfe}

Die Kinder- und Jugendhilfe ist nur eine von vielen kommunalen (Pflicht-) Aufgaben. Ihr Stellenwert hängt zu weiten Teilen von der die kommunale Politik prägende Mehrheitsmeinung in den $\mathrm{Ra}$ thäusern und der politischen Sichtweise und den Präferenzen der politisch verantwortlichen Personen ab. Dabei dürften persönliche Einstellungen mindestens ebenso bedeutsam sein, wie die parteipolitische Zugehörigkeit der verantwortlichen Personen.

Während einzelne Teilbereiche der Kinder- und Jugendhilfe, wie etwa das Angebot an Betreuungsplätzen für Kinder, in- 
zwischen vielerorts wichtige Faktoren für die Attraktivität als Wirtschaftsstandort sind und damit auf größere Aufmerksamkeit hoffen können, wird etwa der Aufwand für Hilfen zur Erziehung - namentlich die stationären Hilfen - vor allem als Kostenbelastung gesehen. Längerfristige volkswirtschaftliche Perspektiven im Hinblick auf die gesellschaftliche Eingliederung junger Menschen bleiben meist unberücksichtigt, weil sie jedenfalls keine kurzfristigen Folgen für die kommunalen Budgets haben.

In den letzten Jahren hat es wiederholt Versuche gegeben, die Gestaltungsspielräume der Kommunen durch eine Streichung von rechtlichen Verpflichtungen zu erweitern, so zuletzt durch den Entwurf eines kommunalen Entlastungsgesetzes (KEG). Dabei ist - wie eine Auswertung der Statistik zeigt - zu Unrecht unterstellt worden, die Ausgaben in der Jugendhilfe wären überproportional zu den Gesamtausgaben der Kommunen gestiegen. Das Gegenteil ist richtig: Während die Gesamtausgaben zwischen 2000 und 2006 um 32,7 Prozent gestiegen sind, nahmen die Ausgaben in der Jugendhilfe nach einer Auswertung der Arbeitsstelle für Kinderund Jugendhilfestatistik an der Universität Dortmund (nur) um 17,7 Prozent zu.

\section{Die Aufgabenwahrnehmung auf der örtlichen Ebene}

Die aktuelle Situation kommunaler Jugendhilfe wird auch geprägt durch die unterschiedliche Ausgestaltung der Aufgabenwahrnehmung auf der örtlichen Ebene. Die Größe und Ausstattung der Jugendämter variiert nicht nur im Hinblick auf den unterschiedlichen Zuschnitt der Kreise und kreisfreien Städte. In vielen Ländern ist eine Tendenz zu beobachten, einzelne Aufgaben der Kinder- und Jugendhilfe, insbesondere im Bereich der Tagesbetreuung aus dem Aufgabenspektrum der (Kreis-) Jugendämter herauszulösen und sie den einzelnen Gemeinden zu übertragen. Durch die Tendenz zur Auslagerung wird die Gesamtzuständigkeit des Jugendamts für alle Aufgaben des örtlichen Trägers entscheidend geschwächt. Synergieeffekte, die mit der Aufgabenkonzentration verbunden sind, gehen verloren.

Noch unübersehbar sind die weitergehenden Folgen der Föderalismusreform I.
So können die Länder nicht nur die Organisation der Jugendämter - namentlich ihre sogenannte Zweigliedrigkeit - ändern. Sie können darüber hinaus das Jugendamt ganz auflösen oder den Kommunen die Befugnis erteilen, Aufgaben aus dem SGB VIII auch anderen Organisationseinheiten zuweisen. Sieht man einmal von der Tendenz ab, die Bezeichnung der Behörde Jugendamt durch Begriffe wie Amt für Jugend und Familie zu erweitern - eine Entwicklung, die bereits seit Jahren zu beobachten ist und im Hinblick auf die Aufgabenstellung durchaus $\mathrm{zu}$ begrüßen ist, so ist im Augenblick noch keine Tendenz zur Zerschlagung der Jugendämter erkennbar.

Vielerorts ist es in den letzten Jahren auch zu einer Neuorganisation der sozialen Dienste (des Jugendamts) gekommen. So wird nicht nur eine Tendenz zum Ab-
16 (Allgemeine Förderung der Erziehung in der Familie) ein erheblicher Gestaltungsspielraum. Zwar kennt auch das Kinderund Jugendhilferecht einklagbare Rechtsansprüche. Da Gegenstand der Leistungen aber in der Regel personenbezogene soziale Dienstleistungen sind, deren Voraussetzungen, Inhalt und Dauer sich - im Gegensatz zu Geldleistungen - nicht unmittelbar aus dem Gesetz ergeben, sondern erst mit Hilfe eines kooperativen Prozesses im konkreten Einzelfall festgestellt werden müssen, bleiben auch hier nicht unerhebliche Gestaltungsspielräume. Minder- oder Schlechtleistungen, die Anlass zur Einlegung von Rechtsbehelfen bieten, werden nicht ohne weiteres erkannt. Abgesehen davon ist in vielen Fällen die Motivation, eine solche Leistung in Anspruch zu nehmen, begrenzt - und damit auch das Interesse, die Leistung notfalls einzuklagen.

\section{»Noch ist keine Tendenz zur Abschaffung der Jugendämter erkennbar - glücklicherweise"}

bau von Spezialdiensten erkennbar, was häufig auch zum Verlust spezifischer Fachkompetenzen führt. Zu erkennen ist darüber hinaus eine Tendenz zur Regionalisierung und Dezentralisierung.

\section{Die begrenzte \\ Steuerungswirkung bundesgesetzlicher Vorgaben}

Bereits aus diesen wenigen Überlegungen lässt sich erahnen, wie unterschiedlich die Rahmenbedingungen für die Erfüllung der Aufgaben der Jugendhilfe in den einzelnen Kreisen und Städten sind und auf welch unterschiedliche Weise deshalb die für alle kommunalen Gebietskörperschaften gleichermaßen geltenden bundesrechtlichen Vorgaben konkret umgesetzt werden.

Hinzu kommt, dass die Steuerungswirkung gesetzlicher Vorgaben im Bereich Sozialer Arbeit begrenzt ist. Zwar sind alle im Gesetz geregelten Aufgaben kommunale Pflichtaufgaben. Aber sie unterscheiden sich zum Teil deutlich im Hinblick auf den Grad der Konkretisierung. So bleibt den Kommunen etwa bei der Erfüllung der Aufgaben nach $\mathbb{\$} 11$ (Jugendarbeit) oder $\mathbb{S}$
Im Fokus der politischen Aufmerksamkeit stehen in den letzten Jahren insbesondere zwei Teilbereiche aus dem Aufgabenspektrum der Kinder- und Jugendhilfe: der Ausbau der Tagesbetreuung für Kinder und der Kinderschutz.

\section{Der Ausbau der Tagesbetreuung für Kinder}

Mehr als zehn Jahre nach der stufenweisen Einführung des Rechtsanspruchs auf einen Kindergartenplatz hat der Gesetzgeber im Hinblick auf die Tagesbetreuung von Kindern in kurzer Folge zwei Gesetze verabschiedet und damit die Kommunen zum bedarfsgerechten Ausbau der Tagesbetreuung für Kinder im Alter unter drei Jahren verpflichtet. Damit soll die bundesweit durchschnittliche Versorgungsquote von derzeit etwa 15,5 Prozent über 21 Prozent bis zum Jahr 2010 aufgrund des Tagesbetreuungsausbaugesetzes (TAG) auf schließlich 35 Prozent bis zum Jahr 2013 durch das im letzten Jahr verabschiedete Kinderförderungsgesetz (KiFöG) angehoben werden. Dies geschieht dadurch, dass in $\$ 24$ SGB VIII 
- bis zum Jahre 2013 die Bedarfskriterien als gesetzliche Vorgaben für die Verpflichtung zum Nachweis eines Betreuungsplatzes erweitert werden und

- ab 2013 die objektiv-rechtliche Verpflichtung im Hinblick auf Kinder, die das erste Lebensjahr vollendet haben, durch einen (einklagbaren) Rechtsanspruch ersetzt wird.

Gegenstand des Anspruchs sind sowohl Plätze in Tageseinrichtungen als auch Plätze in Kindertagespflege. Nachdem die fachlichen Anforderungen an die Tagespflegepersonen bereits durch das Tagesbetreuungsausbaugesetz erhöht worden sind, werden jetzt Signale im Hinblick auf eine bessere Entlohnung (»Leistungsgerechte Vergütung «) gesetzt (\$23) und die sozialversicherungsrechtlichen Folgen der Einkommensteuerpflicht für die Zahlungen des Jugendamts abgemildert (Änderung von SGB V und XI). Das Gesetz setzt auch einen qualitativen Rahmen für die in einigen Ländern etablierte sogenannte Großtagespflege ( $\$ 43$ Abs.3).

Nachholbedarf besteht dabei im Wesentlichen in den westlichen Bundesländern. Das Ausbauvolumen und die Ausbauintensität vor Ort hängen dabei nicht nur von den gesetzlichen Vorschriften, sondern vor allem vom Nachfrageverhalten der Eltern ab. Die Finanzierungslast trifft in erster Linie die Kommunen, darüber hinaus aber auch die Länder. Sie tragen aufgrund des jeweiligen Kommunalverfassungsrechts die Verpflichtung, Mehrbelastungen der Kommunen aufgrund gesetzlicher Aufgabenzuweisungen auszugleichen (Konnexitätsprinzip). Dies gilt seit der Föderalismusreform I auch für Mehrbelastungen der Kommunen, die durch Bundesgesetze verursacht werden. Der Bund darf nämlich künftig den Kommunen keine Aufgaben mehr unmittelbar zuweisen (Art. 84 Abs.1 Satz 7 GG), sondern muss die Bestimmung der für die Ausführung zuständigen Träger den Ländern überlassen. Zu diesem Zweck wurde im Rahmen des Kinderförderungsgesetzes die bundesrechtliche Bestimmung der örtlichen Träger in $\$ 69$ Abs.1 SGB VIII gestrichen. Damit erhält die Bestimmung der örtlichen Träger durch die Länder eine konstitutive Bedeutung. Gleichzeitig hat sich der Bund bereiterklärt, sich an den investiven Kosten für den Ausbau zu beteiligen (Finanzhilfen nach Art. 104 b GG) und darüber hinaus durch Verzicht auf Anteile an der Umsatzsteuer den Ländern im Hinblick auf die steigenden Betriebskosten entgegenzukommen (Änderung des Gesetzes über den Finanzausgleich).

Die Debatte um den Ausbau der Tagesbetreuung wird begleitet durch die Forderung nach einem kostenfreien Zugang zu den Angebotsformen, die für die Kommunen mit Einnahmeverlusten verbunden ist. Das Bundesrecht ermächtigt zur Erhebung von Elternbeiträgen, verpflichtet aber nicht dazu ( $\mathbb{9} 90$ SGB VIII). So variieren die (sozial gestaffelten) Elternbeiträge quer durch die Bundesrepublik nicht nur in der Höhe und der Spreizung der Beitragsstufen erheblich, auch die generelle Abschaffung von Elternbeiträgen schreitet mit ganz unterschiedlicher Geschwindigkeit voran.

\section{Der Schutzauftrag bei Kindeswohlgefährdung}

Das zweite Aufgabenfeld, das derzeit wie kaum ein anderes Schlagzeilen macht, ist der Kinderschutz - angefangen bei der Diskussion über die Verbindlichkeit der Vorsorgeuntersuchungen für Kinder über »Frühe Hilfen « - ein inhaltlich unterschiedlich ausgefüllter Begriff - bis hin zu den (Zwangs-) Befugnissen der Jugendämter im Fall festgestellter Kindeswohlgefährdung. Im Kinder- und Jugendhilfeweiterentwicklungsgesetz (KICK) war mit Wirkung vom 1. Oktober 2005 der bereits bis dahin bestehende Schutzauftrag des Jugendamtes bei Kindeswohlgefährdung strukturiert und konkretisiert und mit den Handlungspflichten freier Träger verknüpft worden (\$ 8a SGB VIII). Auslöser für die aktuelle Diskussion ist aber weniger die Gesetzesänderung als spektakuläre Einzelfälle und deren mediale Aufbereitung. Sehr schnell urteilen die Medien darüber, ob das Jugendamt zur rechten Zeit die richtigen Schritte unternommen hat. Dabei werden die Schwierigkeiten, die mit der Gefährdungseinschätzung verbunden sind, gerne unterschätzt. Die Aufmerksamkeit, die dieses Thema in den letzten Monaten in den Medien erfahren hat, hat dazu geführt, dass die Meldungen über (mögliche) Anzeichen von Kindeswohlgefährdung, die bei den Jugendämtern eingehen, sprunghaft gestiegen sind. Ein Teil davon geht auch auf landesrechtliche Verpflichtungen zurück, das Nichterscheinen von Eltern mit ihrem Kind bei den Vorsorgeuntersuchungen trotz wiederholter Einladung dem Jugendamt zu melden.

Der damit verbundene Aufgabenzuwachs wird unterschiedlich bewältigt. So ist es nicht nur in Berlin, sondern auch in anderen Städten, Großstädten und Kreisen zu zum Teil nicht unerheblichen Personalaufstockungen gekommen. Dabei wird im Auge zu behalten sein, ob die vorgenommenen Stellenaufstockungen auch dann erhalten bleiben, wenn die öffentliche Aufmerksamkeit für dieses Thema nachlässt. An anderen Orten wurde das Problem auf die bekannte Weise bewältigt: durch Umschichtungen zulasten anderer Aufgaben innerhalb und außerhalb der Kinder- und Jugendhilfe. Als Folge der Einführung des $₫ 8 \mathrm{a}$ haben die Jugendämter und Landesjugendämter ein umfassendes Fortbildungsprogramm aufgelegt, um das Personal im Allgemeinen Sozialdienst, aber auch in den Einrichtungen und Diensten freier Träger im Hinblick auf die Gefährdungseinschätzung zu qualifizieren.

Im Hinblick auf den Kinderschutz steht die kommunale Jugendhilfe aber nicht nur vor der Aufgabe, Personal und Organisationsabläufe weiter zu qualifizieren, sondern darüber hinaus die Kooperation mit den anderen Akteuren im Kinderschutz zu verbessern - vom Gesundheitswesen über die die Familiengerichte bis hin zur Polizei. Dabei sind auch die Schulen stärker einzubeziehen. In den Kinderschutzgesetzen einzelner Länder wird die Einrichtung von lokalen Netzwerken, Arbeitskreisen und runder Tische (meist in der Federführung der Jugendämter) verbindlich vorgeschrieben.

\section{Fazit}

Die Erwartungen und Anforderungen an die kommunale Kinder- und Jugendhilfe werden - gerade in den beiden zuletzt skizzierten Aufgabengebieten - weiter steigen. Eine bessere "kommunale Versorgung « in diesen beiden Feldern ist aber nur mit (erheblichen) zusätzlichen Mitteln möglich. Da diese unzureichend bereitgestellt werden und es zu internen Umschichtungen kommt, ist es notwendig, die Aufmerksamkeit immer wieder auf die "vom Aussterben bedrohten Landschaften ", wie die Jugendsozialarbeit oder die Hilfen für Junge Volljährige zu richten und die Erfüllung aller gesetzlichen Aufgaben einzufordern. 